\title{
Existence of solutions for fractional differential equations with integral boundary conditions at resonance
}

\author{
Wei Zhang, Wenbin Liu* \\ Department of Mathematics, China University of Mining and Technology, Xuzhou 221116, P. R. China. \\ Communicated by M. De La Sen
}

\begin{abstract}
This paper investigates the existence of solutions for Riemann-Stieltjes integral boundary value problems of fractional differential equation by using Mawhin's coincidence degree theory. An example is given to show the application of our result. (C)2017 All rights reserved.
\end{abstract}

Keywords: Riemann-Stieltjes integral, fractional differential equations, resonance, coincidence degree. 2010 MSC: 34A08, 34B15.

\section{Introduction}

In recent years, by the extensive development of the theory for fractional calculus, the fractional differential equations have been applied in many research fields, such as physics, chemistry, biology, control theory, economics, biophysics, signal and image processing, etc. (see [6, 8, 10, 13-15]). For example, SIS epidemic can be modeled with fractional derivatives, which is given by

$$
\left\{\begin{array}{l}
D^{\alpha_{1}} S(t)=\Lambda-\beta S I-\mu S+\phi I \\
D^{\alpha_{1}} I(t)=\beta S I-(\phi+\mu+\alpha) I
\end{array}\right.
$$

where $\mathrm{D}^{\alpha_{1}}$ is Caputo fractional derivatives with $0<\alpha_{1} \leqslant 1, S(t)$ is the number of individuals in the susceptible class at time $t$ and $I(t)$ is the number of individuals who are infectious at time $t$ (see [6]). Furthermore, a large number of valuable results about fractional boundary value problems have been achieved by many scholars (see [2-4, 7]). Bai and Lü [3] investigated the following fractional boundary value problems

$$
\left\{\begin{array}{l}
D_{0+}^{\alpha} u(t)+f(t, u(t))=0, \quad 0<t<1 \\
u(0)=u(1)=0
\end{array}\right.
$$

\footnotetext{
*Corresponding author

Email addresses: zhangwei_azyw@163.com (Wei Zhang), cumt_equations@126.com (Wenbin Liu)
} 
where $\mathrm{D}_{0+}^{\alpha}$ is the standard Riemann-Liouville fractional derivative with $1<\alpha \leqslant 2$, and $f \in[0,1] \times$ $[0, \infty) \rightarrow[0, \infty)$ is continuous. By using fixed-point theorems on cone, the existence and multiplicity of positive solutions are obtained.

Recently, there are some papers deal with the existence of solutions for differential equation with Riemann-Stieltjes integral boundary value problems and got some interesting results (see [1, 5, 9, 16-19]). For example, In [5], Cui considered the solvability of second order boundary value problems at resonance involving Riemann-Stieltjes integral conditions by using Mawhin's coincidence degree theory:

$$
\left\{\begin{array}{l}
x^{\prime \prime}(t)=f\left(t, x(t), x^{\prime}(t)\right), \quad t \in(0,1), \\
x(0)=\int_{0}^{1} x(s) d \alpha(s), \quad x(1)=\int_{0}^{1} x(s) d \beta(s),
\end{array}\right.
$$

where $\alpha, \beta$ are functions of bounded variation, $\int_{0}^{1} x(s) d \alpha(s)$ and $\int_{0}^{1} x(s) d \beta(s)$ denote the Riemann-Stieltjes integrals, $f \in \mathrm{C}\left([0,1] \times \mathbb{R}^{2}, \mathbb{R}\right)$.

In [18], Zhang and Han investigated the existence and uniqueness of positive solutions for the following higher order nonlocal fractional differential equations by using monotone iterative technique:

$$
\left\{\begin{array}{l}
D_{0+}^{\alpha} x(t)+f(t, x(t))=0, \quad 0<t<1, \quad n-1<\alpha \leqslant n, \\
x^{(k)}(0)=0, \quad 0 \leqslant k \leqslant n-2, x(1)=\int_{0}^{1} x(s) d A(s),
\end{array}\right.
$$

where $D_{0+}^{\alpha}$ is the standard Riemann-Liouville fractional derivative with $\alpha \geqslant 2, A$ is a function of bounded variation and $\int_{0}^{1} x(s) d A(s)$ denotes the Riemann-Stieltjes integral of $x$ with respect to $A, d A$ can be a signed measure, $f:(0,1) \times \mathbb{R}^{+} \rightarrow \mathbb{R}^{+}$is a continuous function.

Thus, motivated by the results mentioned, in this paper, we discuss the following Riemann-Stieltjes integral boundary value problems by using Mawhin's continuous theorem:

$$
\left\{\begin{array}{l}
D_{0+}^{\alpha} x(t)=f\left(t, x(t), D_{0+}^{\alpha-1} x(t)\right), \quad t \in(0,1), \\
\lim _{t \rightarrow 0^{+}} t^{2-\alpha} x(t)=\int_{0}^{1} x(t) d A(t), \quad x(1)=\int_{0}^{1} x(t) d B(t),
\end{array}\right.
$$

where $D_{0+}^{\alpha}$ is the standard Riemann-Liouville fractional derivative with $1<\alpha \leqslant 2, f:[0,1] \times \mathbb{R}^{2} \rightarrow \mathbb{R}$ is a Carathéodory function, $A$ and $B$ are functions of bounded variation, $\int_{0}^{1} x(t) d A(t)$ and $\int_{0}^{1} x(t) d B(t)$ denote by two Riemann-Stieltjes integrals.

Our innovations can be shown in two points: Firstly, to the best of author's knowledge, there are no papers consider fractional boundary value problem at resonance with Riemann-Stieltjes integral, so our paper enriches some known existing articles. Secondly, our paper extends the result of [5] from integer order differential equation problem to fractional differential equation problem, when we take $\alpha=2$, the result of [5] will be a particular case of our result.

Throughout this paper, we assume that the following condition holds:

(H0) $\wedge_{1} \wedge_{2} \Lambda_{3} \Lambda_{4} \neq 0, \Lambda_{1} \wedge_{4}-\Lambda_{2} \Lambda_{3}=0$ and

$$
\Lambda=\frac{\Lambda_{3}}{\Gamma(\alpha+1)} \int_{0}^{1} t^{\alpha-1}(1-t) d A(t)+\frac{\Lambda_{1}}{\Gamma(\alpha+1)} \int_{0}^{1} t^{\alpha-1}(1-t) d B(t) \neq 0,
$$

where

$$
\begin{aligned}
& \Lambda_{1}=1-\int_{0}^{1} t^{\alpha-2}(1-t) d A(t), \quad \Lambda_{2}=\int_{0}^{1} t^{\alpha-1} d A(t), \\
& \Lambda_{3}=\int_{0}^{1} t^{\alpha-2}(1-t) d B(t), \quad \Lambda_{4}=1-\int_{0}^{1} t^{\alpha-1} d B(t) .
\end{aligned}
$$

A boundary value problem is said to be resonance, if the corresponding homogeneous boundary value problem has a nontrivial solution. Mawhin's continuous theorem [11,12] is an effective tool to solve this 
kind of problem. We note that if condition (H0) holds, then Riemann-Stieltjes integral boundary value problem (1.1) happens to be at resonance in the sense that the following boundary value problem

$$
\left\{\begin{array}{l}
D_{0+}^{\alpha} x(t)=0, \quad t \in(0,1), \\
\lim _{t \rightarrow 0^{+}} t^{2-\alpha} x(t)=\int_{0}^{1} x(t) d A(t), \quad x(1)=\int_{0}^{1} x(t) d B(t),
\end{array}\right.
$$

has $x(t)=c[1+(\rho-1) t] t^{\alpha-2}, c \in \mathbb{R}, \rho=\Lambda_{3} / \Lambda_{4}=\Lambda_{1} / \Lambda_{2}$, as a nontrivial solution.

The structure of this paper is as follows. In Section 2, we recall some definitions and lemmas. In Section 3, based on the Mawhin's continuation theorem, we establish an existence theorem for the problem (1.1). In Section 4, an example is given to illustrate the usefulness of our main results.

\section{Preliminaries}

In this section, we recall some definitions, lemmas which are used throughout this paper.

Let $X$ and $Y$ be two Banach spaces with the norms $\|\cdot\|_{X}$ and $\|\cdot\|_{Y}$, respectively. Define $L: \operatorname{dom}(L) \subset$ $X \rightarrow Y$ be a Fredholm operator with index zero, $P: X \rightarrow X, Q: Y \rightarrow Y$ be two projectors such that

$$
\operatorname{Im} P=\operatorname{KerL}, \quad \operatorname{Im} L=\operatorname{KerQ}, \quad X=\operatorname{KerL} \oplus \operatorname{KerP}, \quad Y=\operatorname{Im} L \oplus \operatorname{Im} Q,
$$

then, $\left.\mathrm{L}\right|_{\text {domLnKerp }}: \operatorname{domL} \rightarrow \operatorname{Im} \mathrm{L}$, is invertible. We denote the inverse by $K_{p}$. Let $\Omega$ be an open bounded subset of $X$ and $\operatorname{dom} L \cap \bar{\Omega} \neq \emptyset$, then the map $N: X \rightarrow Y$ is called L-compact on $\bar{\Omega}$, if $Q N(\bar{\Omega})$ is bounded and $K_{P, Q} N=K_{p}(I-Q) N: \bar{\Omega} \rightarrow X$ is compact (see [11, 12]).

Lemma $2.1([11,12])$. Let $\mathrm{L}: \operatorname{dom} \mathrm{L} \subset \mathrm{X} \rightarrow \mathrm{Y}$ be a Fredholm operator of index zero and $\mathrm{N}: \mathrm{X} \rightarrow \mathrm{Y}$ is L-compact on $\bar{\Omega}$. Assume that the following conditions are satisfied:

(i) $\mathrm{Lu} \neq \lambda \mathrm{Nu}$ for any $\mathrm{u} \in(\operatorname{dom} \mathrm{L} \backslash \operatorname{KerL}) \cap \partial \Omega, \lambda \in(0,1)$;

(ii) $\mathrm{Nu} \notin \operatorname{Im} \mathrm{L}$ for any $\mathrm{u} \in \operatorname{Ker} \mathrm{L} \cap \partial \Omega$;

(iii) $\operatorname{deg}\left(\left.\mathrm{QN}\right|_{\text {KerL }}, \Omega \cap \operatorname{Ker} \mathrm{L}, 0\right) \neq 0$.

Then the equation $\mathrm{Lx}=\mathrm{N} x$ has at least one solution in dom $\mathrm{L} \cap \bar{\Omega}$.

Definition $2.2([8,13])$. The Rieman-Liouville fractional integral of order $\alpha>0$ for function $x:(0,+\infty) \rightarrow$ $\mathbb{R}$ is given by

$$
\mathrm{I}_{0+}^{\alpha} x(\mathrm{t})=\frac{1}{\Gamma(\alpha)} \int_{0}^{t}(t-s)^{\alpha-1} x(s) d s,
$$

provided the right side integral is pointwise defined on $(0,+\infty)$.

Definition 2.3 ([8, 13]). The Riemann-Liouville fractional derivative of order $\alpha>0$ for function $x$ : $(0,+\infty) \rightarrow \mathbb{R}$ is given by

$$
D_{0+}^{\alpha} x(t)=\frac{d^{n}}{d t^{n}} I_{0+}^{n-\alpha} x(t)=\frac{1}{\Gamma(n-\alpha)} \frac{d^{n}}{d t^{n}} \int_{0}^{t}(t-s)^{n-\alpha-1} x(s) d s
$$

where $n=[\alpha]+1$ provided the right side integral is pointwise defined on $(0,+\infty)$.

Lemma $2.4([3,7,8,13])$. Let $\alpha>0$. Assume that $x, \mathrm{D}_{0+}^{\alpha} x \in \mathrm{L}^{1}(0,1)$, then the following equality holds

$$
\mathrm{I}_{0+}^{\alpha} \mathrm{D}_{0+}^{\alpha} x(\mathrm{t})=x(\mathrm{t})+\mathrm{c}_{1} \mathrm{t}^{\alpha-1}+\mathrm{c}_{2} \mathrm{t}^{\alpha-2}+\cdots+\mathrm{c}_{\mathrm{n}} \mathrm{t}^{\alpha-n},
$$

where $\mathrm{n}=[\alpha]+1, \mathrm{c}_{\boldsymbol{i}} \in \mathbb{R}, \boldsymbol{i}=1,2, \cdots, \mathrm{n}$. 
Lemma 2.5 ([3, 7, 8, 13]). Assume that $x \in \mathrm{L}^{1}(0,1), \alpha \geqslant \beta \geqslant 0$, then

$$
I_{0+}^{\alpha} I_{0+}^{\beta} x(t)=I_{0+}^{\alpha+\beta} x(t), \quad D_{0+}^{\beta} I_{0+}^{\alpha} x(t)=I_{0+}^{\alpha-\beta} x(t) .
$$

Lemma $2.6([3,7])$. Assume that $\alpha>0, \lambda>-1, t>0$, then

$$
\mathrm{I}_{0+}^{\alpha} \mathrm{t}^{\lambda}=\frac{\Gamma(\lambda+1)}{\Gamma(\lambda+1+\alpha)} \mathrm{t}^{\alpha+\lambda}, \quad \mathrm{D}_{0+}^{\alpha} \mathrm{t}^{\lambda}=\frac{\Gamma(\lambda+1)}{\Gamma(\lambda+1-\alpha)} \mathrm{t}^{\lambda-\alpha},
$$

in particular $\mathrm{D}_{0+}^{\alpha} \mathrm{t}^{\alpha-\mathrm{m}}=0, \mathrm{~m}=1,2, \cdots, \mathrm{n}$, where $\mathrm{n}=[\alpha]+1$.

\section{Main result}

In this part, we let $x_{\alpha}(t)=t^{2-\alpha} x(t)$ and take

$$
X=\left\{x: x_{\alpha}, D_{0+}^{\alpha-1} x \in C[0,1]\right\}, \quad Y=L^{1}[0,1] .
$$

It is easy to check that $X$ and $Y$ are two Banach spaces with norms

$$
\|x\|=\max \left\{\left\|x_{\alpha}\right\|_{\infty},\left\|D_{0+}^{\alpha-1} x\right\|_{\infty}\right\},\|y\|=\|y\|_{1}=\int_{0}^{1}|y(t)| d t,
$$

respectively, where $\|x\|_{\infty}=\sup _{t \in[0,1]}|x(t)|$.

Define the linear operator $\mathrm{L}: \operatorname{dom} \mathrm{L} \subset \mathrm{X} \rightarrow \mathrm{Y}$ and nonlinear operator $\mathrm{N}: \mathrm{X} \rightarrow \mathrm{Y}$ as follows:

$$
L x(t)=D_{0+}^{\alpha} x(t), x(t) \in \operatorname{dom} L, \quad N x(t)=f\left(t, x(t), D_{0+}^{\alpha-1} x(t)\right), x(t) \in X,
$$

where

$$
\operatorname{dom} L=\left\{x \in X: D_{0+}^{\alpha} x(t) \in Y, x \text { satisfies boundary value conditions of (1.1) }\right\} .
$$

Then problem (1.1) is equivalent to the operator equation $L x=N x, x \in$ domL.

Lemma 3.1. Assume that ( $\mathrm{H} 0)$ holds. Then operator $\mathrm{L}: \operatorname{dom} \mathrm{L} \subset \mathrm{X} \rightarrow \mathrm{Y}$ satisfies,

$$
\begin{array}{r}
\operatorname{Ker} L=\left\{x \in \operatorname{dom} L: x(t)=c[1+(\rho-1) t] t^{\alpha-2}, c \in \mathbb{R}\right\}, \\
\operatorname{ImL}=\left\{y \in Y: \Lambda_{3} \int_{0}^{1} \int_{0}^{1} g(t, s) y(s) d s d A(t)\right. \\
\left.+\Lambda_{1} \int_{0}^{1} \int_{0}^{1} g(t, s) y(s) d s d B(t)=0\right\},
\end{array}
$$

where $\rho=\Lambda_{3} / \Lambda_{4}=\Lambda_{1} / \Lambda_{2}$, and

$$
g(t, s)=\frac{1}{\Gamma(\alpha)}\left\{\begin{array}{l}
t^{\alpha-1}(1-s)^{\alpha-1}, 0 \leqslant t \leqslant s \leqslant 1 \\
t^{\alpha-1}(1-s)^{\alpha-1}-(t-s)^{\alpha-1}, 0 \leqslant s \leqslant t \leqslant 1 .
\end{array}\right.
$$

Proof. If $\mathrm{L} x=\mathrm{D}_{0+}^{\alpha} x=0$, by Lemma 2.4, we have

$$
x(t)=a t^{\alpha-1}+b t^{\alpha-2}, \quad a, b \in \mathbb{R},
$$

which together with boundary conditions of (1.1), we can derive

$$
b=\int_{0}^{1} x(t) d A(t)=\int_{0}^{1}\left(a t^{\alpha-1}+b t^{\alpha-2}\right) d A(t)=a \Lambda_{2}+b\left(\Lambda_{2}-\Lambda_{1}+1\right),
$$


and

$$
a+b=\int_{0}^{1} x(t) d B(t)=\int_{0}^{1}\left(a t^{\alpha-1}+b t^{\alpha-2}\right) d B(t)=a\left(1-\Lambda_{4}\right)+b\left(\Lambda_{3}-\Lambda_{4}+1\right) .
$$

Then, $a=b(\rho-1)$. So, KerL $\subset\left\{x \in \operatorname{domL}: x(t)=c[1+(\rho-1) t] t^{\alpha-2}, c \in \mathbb{R}\right\}$. Conversely, take $x(t)=[1+(\rho-1) t] t^{\alpha-2}$, then $D_{0+}^{\alpha} x=0$ and

$$
\begin{aligned}
\int_{0}^{1} x(t) d A(t) & =\int_{0}^{1} t^{\alpha-2} d A(t)+\int_{0}^{1}(\rho-1) t^{\alpha-1} d A(t) \\
& =1-\Lambda_{1}+\rho \Lambda_{2}=1=\lim _{t \rightarrow 0^{+}} t^{2-\alpha} x(t) \\
\int_{0}^{1} x(t) d B(t) & =\int_{0}^{1} t^{\alpha-2} d B(t)+\int_{0}^{1}(\rho-1) t^{\alpha-1} d B(t) \\
& =\Lambda_{3}+\rho\left(1-\Lambda_{4}\right)=\rho=x(1) .
\end{aligned}
$$

So, $\left\{x \in \operatorname{domL}: x(t)=c[1+(\rho-1) t] t^{\alpha-2}, c \in \mathbb{R}\right\} \subset$ KerL. For $y \in \operatorname{ImL}$, there exists $x \in \operatorname{domL}$ such that $D_{0+}^{\alpha} x(t)=y(t)$. Considering the boundary conditions of (1.1), one has

$$
x(t)=-\int_{0}^{1} g(t, s) y(s) d s+\left[\lim _{t \rightarrow 0^{+}} t^{2-\alpha} x(t)\right](1-t) t^{\alpha-2}+x(1) t^{\alpha-1}
$$

Integrating (3.2) with respect to $d A(t)$ and $d B(t)$ from 0 to 1 , respectively, we obtain

$$
\int_{0}^{1} x(t) d A(t)=-\int_{0}^{1} \int_{0}^{1} g(t, s) y(s) d s d A(t)+\left[\lim _{t \rightarrow 0^{+}} t^{2-\alpha} x(t)\right]\left(1-\Lambda_{1}\right)+x(1) \Lambda_{2},
$$

and

$$
\int_{0}^{1} x(t) d B(t)=-\int_{0}^{1} \int_{0}^{1} g(t, s) y(s) d s d B(t)+\left[\lim _{t \rightarrow 0^{+}} t^{2-\alpha} x(t)\right] \Lambda_{3}+x(1)\left(1-\Lambda_{4}\right) .
$$

By simple calculation, we get

$$
-\frac{\Lambda_{1}}{\Lambda_{3}}=-\frac{\Lambda_{2}}{\Lambda_{4}}=\frac{\int_{0}^{1} \int_{0}^{1} g(t, s) y(s) d s d A(t)}{\int_{0}^{1} \int_{0}^{1} g(t, s) y(s) d s d B(t)}
$$

that is,

$$
\operatorname{ImL} \subset\left\{y \in Y: \Lambda_{3} \int_{0}^{1} \int_{0}^{1} g(t, s) y(s) d s d A(t)+\Lambda_{1} \int_{0}^{1} \int_{0}^{1} g(t, s) y(s) d s d B(t)=0\right\} .
$$

Conversely, let $y \in Y$ satisfy (3.3), take

$$
x(t)=-\int_{0}^{1} g(t, s) y(s) d s-\frac{t^{\alpha-1}}{\Lambda_{4}} \int_{0}^{1} \int_{0}^{1} g(t, s) y(s) d s d B(t),
$$

thus,

$$
\mathrm{Lx}(\mathrm{t})=\mathrm{D}_{0+}^{\alpha} x(\mathrm{t})=\mathrm{y}(\mathrm{t}), \lim _{\mathrm{t} \rightarrow 0^{+}} \mathrm{t}^{2-\alpha} \chi(\mathrm{t})=0,
$$

and

$$
\chi(1)=-\frac{1}{\Lambda_{4}} \int_{0}^{1} \int_{0}^{1} g(t, s) y(s) d s d B(t)
$$

Then, we have

$$
\int_{0}^{1} x(t) d A(t)=-\int_{0}^{1} \int_{0}^{1} g(t, s) y(s) d s d A(t)-\frac{\Lambda_{2}}{\Lambda_{4}} \int_{0}^{1} \int_{0}^{1} g(t, s) y(s) d s d B(t)=0,
$$


and

$$
\begin{aligned}
\int_{0}^{1} x(t) d B(t) & =-\int_{0}^{1} \int_{0}^{1} g(t, s) y(s) d s d B(t)-\frac{1-\Lambda_{4}}{\Lambda_{4}} \int_{0}^{1} \int_{0}^{1} g(t, s) y(s) d s d B(t) \\
& =-\frac{1}{\Lambda_{4}} \int_{0}^{1} \int_{0}^{1} g(t, s) y(s) d s d B(t)=x(1) .
\end{aligned}
$$

Therefore,

$$
\left\{y \in Y: \Lambda_{3} \int_{0}^{1} \int_{0}^{1} g(t, s) y(s) d s d A(t)+\Lambda_{1} \int_{0}^{1} \int_{0}^{1} g(t, s) y(s) d s d B(t)=0\right\} \subset \operatorname{ImL} .
$$

So, (3.1) is satisfied.

Lemma 3.2. Assume that (H0) holds, then $\mathrm{L}$ is a Fredholm operator of index zero. The linear projector operator $\mathrm{P}: \mathrm{X} \rightarrow \mathrm{X}$ and $\mathrm{Q}: \mathrm{Y} \rightarrow \mathrm{Y}$ can be defined by

$$
\begin{aligned}
& (P x)(t)=\left[\lim _{t \rightarrow 0^{+}} t^{2-\alpha} x(t)\right][1+(\rho-1) t] t^{\alpha-2} \\
& (Q y)(t)=\frac{1}{\Lambda}\left[\Lambda_{3} \int_{0}^{1} \int_{0}^{1} g(t, s) y(s) d s d A(t)+\Lambda_{1} \int_{0}^{1} \int_{0}^{1} g(t, s) y(s) d s d B(t)\right] .
\end{aligned}
$$

Proof. Obviously, ImP $=$ KerL. For $x \in X$ we have

$$
\begin{aligned}
\left(P^{2} x\right)(t) & =P(P x(t))=\left[\lim _{t \rightarrow 0^{+}} t^{2-\alpha} P x(t)\right][1+(\rho-1) t] t^{\alpha-2} \\
& =\left[\lim _{t \rightarrow 0^{+}} t^{2-\alpha} x(t)\right][1+(\rho-1) t] t^{\alpha-2}=(P x)(t),
\end{aligned}
$$

and

$$
\begin{aligned}
D_{0+}^{\alpha-1} P x(t) & =\frac{1}{\Gamma(2-\alpha)} \frac{d}{d t} \int_{0}^{t}(t-s)^{1-\alpha}\left[\lim _{t \rightarrow 0^{+}} t^{2-\alpha} x(t) s^{\alpha-2}+\lim _{t \rightarrow 0^{+}} t^{2-\alpha} \chi(t)(\rho-1) s^{\alpha-1}\right] d s \\
& =\frac{1}{\Gamma(2-\alpha)} \frac{d}{d t}\left[\lim _{t \rightarrow 0^{+}} t^{2-\alpha} \chi(t) \Gamma(2-\alpha) \Gamma(\alpha-1)+\lim _{t \rightarrow 0^{+}} t^{2-\alpha} \chi(t)(\rho-1) \Gamma(2-\alpha) \Gamma(\alpha) t\right] \\
& =(\rho-1) \Gamma(\alpha) \lim _{t \rightarrow 0^{+}} t^{2-\alpha} \chi(t) .
\end{aligned}
$$

Hence, $\mathrm{P}: \mathrm{X} \rightarrow \mathrm{X}$ is a continuous linear operator. It follows from $\mathrm{x}=(\mathrm{x}-\mathrm{P} x)+\mathrm{P} x$ that $\mathrm{X}=\mathrm{KerP}+\mathrm{KerL}$. For $x \in \operatorname{KerL} \cap \operatorname{KerP}$, that is, $x \in \operatorname{KerL}$ and $x \in \operatorname{KerP}$, then $x$ can be rewritten as

$$
x(t)=c[1+(\rho-1) t] t^{\alpha-2}, c \in \mathbb{R},
$$

and

$$
0=(P x)(t)=c \lim _{t \rightarrow 0^{+}} t^{2-\alpha}[1+(\rho-1) t] t^{\alpha-2}=c .
$$

So, $\operatorname{KerL} \cap \operatorname{KerP}=\{0\}$. Thus, $X=\operatorname{KerP} \oplus \operatorname{KerL}$. For $y \in Y$, we have

$$
\begin{aligned}
\left(Q^{2} y\right)(t)=Q(Q y(t)) & =\frac{1}{\Lambda}\left[\Lambda_{3} Q y(t) \int_{0}^{1} \int_{0}^{1} g(t, s) d s d A(t)+\Lambda_{1} Q y(t) \int_{0}^{1} \int_{0}^{1} g(t, s) d s d B(t)\right] \\
& =\frac{1}{\Lambda}\left[\frac{\Lambda_{3}}{\Gamma(\alpha+1)} \int_{0}^{1} t^{\alpha-1}(1-t) d A(t)+\frac{\Lambda_{1}}{\Gamma(\alpha+1)} \int_{0}^{1} t^{\alpha-1}(1-t) d B(t)\right] Q y(t) \\
& =Q y(t),
\end{aligned}
$$


which implies that $Q$ is a projector operator. Obviously, $\operatorname{ImL}=$ KerQ. Set $y=(y-Q y)+Q y$, then $(y-Q y) \in \operatorname{KerQ}=\operatorname{ImL}, Q y \in \operatorname{ImQ}$. So, $y=\operatorname{ImL}+\operatorname{ImQ}$. Furthermore, it follows from KerQ $=\operatorname{ImL}$ and $\mathrm{Q}^{2} \mathrm{y}=\mathrm{Q} y$ that $\operatorname{ImL} \cap \operatorname{Im} Q=\{0\}$. Thus, $\mathrm{Y}=\operatorname{ImL} \oplus \operatorname{ImQ}$. Therefore, we have

$$
\operatorname{dim} \operatorname{KerL}=\operatorname{dim} \operatorname{Im} Q=\operatorname{codim} \operatorname{ImL}=1 .
$$

That is, $\mathrm{L}$ is a Fredholm operator of index zero.

Lemma 3.3. Assume that (H0) holds, define linear operator $\mathrm{K}_{\mathrm{p}}: \operatorname{ImL} \rightarrow \operatorname{dom} \mathrm{L} \cap \operatorname{Ker} \mathrm{P}$ by

$$
\left(K_{p} y\right)(t)=-\int_{0}^{1} g(t, s) y(s) d s-\frac{t^{\alpha-1}}{\Lambda_{4}} \int_{0}^{1} \int_{0}^{1} g(t, s) y(s) d s d B(t)
$$

then $\mathrm{K}_{\mathrm{p}}$ is the inverse of $\left.\mathrm{L}\right|_{\text {dom } \mathrm{L} \cap \operatorname{Kerp}}$ and $\left\|\mathrm{K}_{\mathrm{p}} \mathrm{y}\right\|_{\mathrm{X}} \leqslant \Delta\|\mathrm{y}\|_{1}$, for all $\mathrm{y} \in \operatorname{ImL}$, where $\Delta=\frac{2}{\Gamma(\alpha)}+\frac{\left|\int_{0}^{1} \mathrm{t}^{\alpha-1} \mathrm{~d}\right| \mathrm{B}(\mathrm{t})||}{\Gamma(\alpha)\left|\Lambda_{4}\right|}$. Proof. For $y \in \operatorname{ImL}$, we have

$$
\begin{aligned}
\int_{0}^{1}\left(K_{p} y\right)(t) d A(t) & =-\int_{0}^{1} \int_{0}^{1} g(t, s) y(s) d s d A(t)-\frac{\Lambda_{2}}{\Lambda_{4}} \int_{0}^{1} \int_{0}^{1} g(t, s) y(s) d s d B(t) \\
& =-\int_{0}^{1} \int_{0}^{1} g(t, s) y(s) d s d A(t)-\frac{\Lambda_{1}}{\Lambda_{3}} \int_{0}^{1} \int_{0}^{1} g(t, s) y(s) d s d B(t) \\
& =0=\lim _{t \rightarrow 0^{+}} t^{2-\alpha}\left(K_{p} y\right)(t)
\end{aligned}
$$

and

$$
\begin{aligned}
\int_{0}^{1}\left(K_{p} y\right)(t) d B(t) & =-\int_{0}^{1} \int_{0}^{1} g(t, s) y(s) d s d B(t)-\frac{1-\Lambda_{4}}{\Lambda_{4}} \int_{0}^{1} \int_{0}^{1} g(t, s) y(s) d s d B(t) \\
& =-\frac{1}{\Lambda_{4}} \int_{0}^{1} \int_{0}^{1} g(t, s) y(s) d s d B(t)=\left(K_{p} y\right)(1) .
\end{aligned}
$$

So, $K_{p}$ is well-defined on ImL. In addition,

$$
\begin{aligned}
\left(\operatorname{LK}_{p}\right) y(t) & =D_{0+}^{\alpha}\left[-\int_{0}^{1} g(t, s) y(s) d s-\frac{t^{\alpha-1}}{\Lambda_{4}} \int_{0}^{1} \int_{0}^{1} g(t, s) y(s) d s d B(t)\right] \\
& =D_{0+}^{\alpha}\left[-\int_{0}^{1} g(t, s) y(s) d s\right]=y(t) .
\end{aligned}
$$

Furthermore, for $x \in \operatorname{dom} L \cap \operatorname{KerP}$, we have $\lim _{t \rightarrow 0^{+}} t^{2-\alpha} x(t)=0$ and $x(1)=\int_{0}^{1} x(t) d B(t)$, then

$$
\begin{aligned}
\left(K_{p} L\right) x(t)= & -\int_{0}^{1} g(t, s) D_{0+}^{\alpha} x(s) d s-\frac{t^{\alpha-1}}{\Lambda_{4}} \int_{0}^{1} \int_{0}^{1} g(t, s) D_{0+}^{\alpha} x(s) d s d B(t) \\
= & x(t)-\left[\lim _{t \rightarrow 0^{+}} t^{2-\alpha} x(t)\right](1-t) t^{\alpha-2}-x(1) t^{\alpha-1} \\
& +\frac{t^{\alpha-1}}{\Lambda_{4}} \int_{0}^{1}\left\{x(t)-\left[\lim _{t \rightarrow 0^{+}} t^{2-\alpha} x(t)\right](1-t) t^{\alpha-2}-x(1) t^{\alpha-1}\right\} d B(t) \\
= & x(t) .
\end{aligned}
$$

That is, $\mathrm{K}_{\mathrm{p}}=\left(\left.\mathrm{L}\right|_{\text {domL } \cap \text { Kerp }}\right)^{-1}$. Next, we divided $\left\|\mathrm{K}_{\mathrm{p}} \mathrm{y}\right\|_{\mathrm{X}} \leqslant \Delta\|\mathrm{y}\|_{1}$. In fact,

$$
\left\|\mathrm{t}^{2-\alpha} \mathrm{K}_{\mathrm{p}} \mathrm{y}\right\|_{\infty} \leqslant \frac{2}{\Gamma(\alpha)} \int_{0}^{1}|\mathrm{y}(\mathrm{s})| \mathrm{d} s+\frac{1}{\left|\Lambda_{4}\right|}\left|\int_{0}^{1} \int_{0}^{1} \mathrm{~g}(\mathrm{t}, \mathrm{s}) \mathrm{y}(\mathrm{s}) \mathrm{d} \mathrm{d} \mathrm{dB}(\mathrm{t})\right|
$$




$$
\begin{aligned}
& \leqslant \frac{2}{\Gamma(\alpha)}\|y\|_{1}+\frac{1}{\Gamma(\alpha)\left|\Lambda_{4}\right|}\|y\|_{1}\left|\int_{0}^{1} t^{\alpha-1} \mathrm{~d}\right| \mathrm{B}(\mathrm{t})|| \\
& =\Delta\|y\|_{1},
\end{aligned}
$$

and

$$
\begin{aligned}
\left\|D_{0+}^{\alpha-1} K_{p} y\right\|_{\infty} & \leqslant 2 \int_{0}^{1}|y(s)| d s+\frac{\Gamma(\alpha)}{\left|\Lambda_{4}\right|}\left|\int_{0}^{1} \int_{0}^{1} g(t, s) y(s) d s d B(t)\right| \\
& \leqslant 2\|y\|_{1}+\frac{1}{\left|\Lambda_{4}\right|}\|y\|_{1}\left|\int_{0}^{1} t^{\alpha-1} \mathrm{~d}\right| \mathrm{B}(\mathrm{t})|| \\
& \leqslant \frac{2}{\Gamma(\alpha)}\|y\|_{1}+\frac{1}{\Gamma(\alpha)\left|\Lambda_{4}\right|}\|y\|_{1}\left|\int_{0}^{1} t^{\alpha-1} \mathrm{~d}\right| \mathrm{B}(\mathrm{t})|| \\
& =\Delta\|y\|_{1} .
\end{aligned}
$$

Therefore, $\left\|\mathrm{K}_{\mathrm{p}} \mathrm{y}\right\|_{\mathrm{X}} \leqslant \Delta\|\mathrm{y}\|_{1}$.

Lemma 3.4. Assume that (H0) holds and $\Omega \subset X$ is an open bounded subset with dom $\mathrm{L} \cap \bar{\Omega} \neq \emptyset$, then $\mathrm{N}$ is L-compact on $\bar{\Omega}$.

Proof. From $f:[0,1] \times \mathbb{R}^{2} \rightarrow \mathbb{R}$ satisfies the Carathéodory conditions, we can get that $\mathrm{QN}(\bar{\Omega})$ and $(\mathrm{I}-\mathrm{Q}) \mathrm{N}(\bar{\Omega})$ are bounded, that is, there exist constants $\tilde{\mathrm{L}}, \mathrm{L}>0$ such that

$$
|Q N x| \leqslant \tilde{L},|(I-Q) N x| \leqslant L, x \in \bar{\Omega}, \text { a.e. } t \in[0,1] .
$$

So, we only need to show that $\mathrm{K}_{\mathrm{p}}(\mathrm{I}-\mathrm{Q}) \mathrm{N}: \bar{\Omega} \rightarrow \mathrm{X}$ is compact. By Lemma 3.3, $\mathrm{K}_{\mathrm{p}}(\mathrm{I}-\mathrm{Q}) \mathrm{N}(\bar{\Omega})$ is bounded. It follows from the Lebesgue dominated convergence theorem that $K_{p}(I-Q) N: \bar{\Omega} \rightarrow X$ is continuous. For $0 \leqslant t_{1}<t_{2} \leqslant 1, x \in \bar{\Omega}$, we have,

$$
\begin{aligned}
& \left|t_{1}^{2-\alpha} K_{p}(I-Q) N x\left(t_{1}\right)-t_{2}^{2-\alpha} K_{p}(I-Q) N x\left(t_{2}\right)\right| \\
& =\mid-t_{1}^{2-\alpha} \int_{0}^{1} g\left(t_{1}, s\right)(I-Q) N x(s) d s-\frac{t_{1}}{\Lambda_{4}} \int_{0}^{1} \int_{0}^{1} g(t, s)(I-Q) N x(s) d s d B(t) \\
& \quad+t_{2}^{2-\alpha} \int_{0}^{1} g\left(t_{2}, s\right)(I-Q) N x(s) d s+\frac{t_{2}}{\Lambda_{4}} \int_{0}^{1} \int_{0}^{1} g(t, s)(I-Q) N x(s) d s d B(t) \mid \\
& \leqslant \frac{\left(t_{2}-t_{1}\right)}{\left|\Lambda_{4}\right|}\left|\int_{0}^{1} \int_{0}^{1} g(t, s)(I-Q) N x(s) d s d B(t)\right|+\mid \int_{0}^{1} t_{2}^{2-\alpha} g\left(t_{2}, s\right)(I-Q) N x(s) d s \\
& \quad-\int_{0}^{1} t_{1}^{2-\alpha} g\left(t_{1}, s\right)(I-Q) N x(s) d s \mid
\end{aligned}
$$

considering that,

$$
\begin{aligned}
& \left|\int_{0}^{1} t_{2}^{2-\alpha} g\left(t_{2}, s\right)(I-Q) N x(s) d s-\int_{0}^{1} t_{1}^{2-\alpha} g\left(t_{1}, s\right)(I-Q) N x(s) d s\right| \\
& =\frac{1}{\Gamma(\alpha)} \mid \int_{0}^{1} t_{2}(1-s)^{\alpha-1}(I-Q) N x(s) d s-\int_{0}^{t_{2}} t_{2}^{2-\alpha}\left(t_{2}-s\right)^{\alpha-1}(I-Q) N x(s) d s \\
& \quad-\int_{0}^{1} t_{1}(1-s)^{\alpha-1}(I-Q) N x(s) d s+\int_{0}^{t_{1}} t_{1}^{2-\alpha}\left(t_{1}-s\right)^{\alpha-1}(I-Q) N x(s) d s \mid
\end{aligned}
$$




$$
\begin{aligned}
& \leqslant \frac{L}{\Gamma(\alpha)}\left(t_{2}-t_{1}\right)+\mid \int_{0}^{t_{2}} t_{2}^{2-\alpha}\left(t_{2}-s\right)^{\alpha-1}(I-Q) N x(s) d s \\
& \quad-\int_{0}^{t_{1}} t_{1}^{2-\alpha}\left(t_{1}-s\right)^{\alpha-1}(I-Q) N x(s) d s \mid
\end{aligned}
$$

and

$$
\begin{aligned}
\left|\int_{0}^{t_{2}} t_{2}^{2-\alpha}\left(t_{2}-s\right)^{\alpha-1}(I-Q) N x(s) d s-\int_{0}^{t_{1}} t_{1}^{2-\alpha}\left(t_{1}-s\right)^{\alpha-1}(I-Q) N x(s) d s\right| \\
=\mid \int_{t_{1}}^{t_{2}} t_{2}^{2-\alpha}\left(t_{2}-s\right)^{\alpha-1}(I-Q) N x(s) d s \\
\quad+\int_{0}^{t_{1}}\left[t_{2}^{2-\alpha}\left(t_{2}-s\right)^{\alpha-1}-t_{1}^{2-\alpha}\left(t_{1}-s\right)^{\alpha-1}\right](I-Q) N x(s) d s \mid \\
\leqslant \frac{L}{\alpha} t_{2}^{2-\alpha}\left(t_{2}-t_{1}\right)^{\alpha}+\frac{L}{\alpha}\left[\left(t_{2}^{2}-t_{1}^{2}\right)-t_{2}^{2-\alpha}\left(t_{2}-t_{1}\right)^{\alpha}\right] .
\end{aligned}
$$

Since $t$ and $t^{2}$ are uniformly continuous on $[0,1]$, we can get $t^{2-\alpha} K_{p}(I-Q) N(\bar{\Omega})$ is equicontinuous. In addition,

$$
\begin{gathered}
\left|\mathrm{D}_{0+}^{\alpha-1} \mathrm{~K}_{\mathrm{p}}(\mathrm{I}-\mathrm{Q}) \mathrm{N} x\left(\mathrm{t}_{1}\right)-\mathrm{D}_{0+}^{\alpha-1} \mathrm{~K}_{\mathrm{p}}(\mathrm{I}-\mathrm{Q}) \mathrm{N} x\left(\mathrm{t}_{2}\right)\right| \\
=\left|\int_{\mathrm{t}_{1}}^{\mathrm{t}_{2}}(\mathrm{I}-\mathrm{Q}) \mathrm{N} x(\mathrm{~s}) \mathrm{ds}\right| \leqslant \mathrm{L}\left(\mathrm{t}_{2}-\mathrm{t}_{1}\right) .
\end{gathered}
$$

Since $t$ is uniformly continuous on $[0,1]$, we can get $D_{0+}^{\alpha-1} K_{p}(I-Q) N(\bar{\Omega})$ is equicontinuous. By ArzelàAscoli theorem, we obtain that $\mathrm{K}_{\mathrm{p}}(\mathrm{I}-\mathrm{Q}) \mathrm{N}: \bar{\Omega} \rightarrow \mathrm{X}$ is compact.

In order to obtain our main results, we suppose that the following conditions are satisfied:

(H1) There exist nonnegative functions $p, q, r \in Y$ such that

$$
|f(t, x, y)| \leqslant t^{2-\alpha} p(t)|x|+q(t)|y|+r(t), \quad \forall(t, x, y) \in[0,1] \times \mathbb{R}^{2},
$$

and

$$
\|p\|_{1}+\|q\|_{1}<\frac{\Gamma(\alpha)}{4 \delta+\Gamma(\alpha) \Delta}
$$

(H2) There exists a constant $G>0$ such that if $t^{2-\alpha}|x(t)|>G$ or $\left|D_{0+}^{\alpha-1} \chi(t)\right|>G$ for all $t \in(0,1)$, then

$$
\mathrm{QN}(x(\mathrm{t})) \neq 0 \text {. }
$$

(H3) There exists a constant $M>0$ such that for all $c \in \mathbb{R}$, if $|c|>M$, then either

$$
\mathrm{cQN}\left\{\mathrm{c}[1+(\rho-1) \mathrm{t}] \mathrm{t}^{\alpha-2}\right\}>0,
$$

or

$$
\mathrm{cQN}\left\{\mathrm{c}[1+(\rho-1) t] \mathrm{t}^{\alpha-2}\right\}<0 .
$$

Lemma 3.5. Suppose that (H1) and (H2) hold, set

$$
\Omega_{1}=\{x \in \operatorname{dom} \mathrm{L} \backslash \operatorname{Ker} \mathrm{L}: \mathrm{Lx}=\lambda \mathrm{N} x, \lambda \in(0,1)\},
$$

note $\delta=\max \{1,|\rho|,|\rho-1|\}$. Then $\Omega_{1}$ is bounded.

Proof. For $x \in \Omega_{1}$, we have $N x \in \operatorname{ImL}$, that is, $\mathrm{QN}(x(\mathrm{t}))=0$. Thus, from (H2), we obtain that there exist 
constants $t_{0}, t_{1} \in(0,1)$ such that $t_{0}^{2-\alpha}\left|x\left(t_{0}\right)\right| \leqslant G$ and $\left|D_{0+}^{\alpha-1} \chi\left(t_{1}\right)\right| \leqslant G$. It follows from $L x=\lambda N x$ that

$$
x(t)=\lambda I_{0+}^{\alpha} f\left(t, x(t), D_{0+}^{\alpha-1} x(t)\right)+c_{1} t^{\alpha-1}+c_{2} t^{\alpha-2}, \quad c_{1}, c_{2} \in \mathbb{R} .
$$

Then,

$$
D_{0+}^{\alpha-1} \chi(t)=\lambda \int_{0}^{t} f\left(s, x(s), D_{0+}^{\alpha-1} \chi(s)\right) d s+c_{1} \Gamma(\alpha)
$$

and

$$
t^{2-\alpha} \chi(t)=\lambda t^{2-\alpha} I_{0+}^{\alpha} f\left(t, x(t), D_{0+}^{\alpha-1} \chi(t)\right)+c_{1} t+c_{2}
$$

So,

$$
\begin{aligned}
\left|c_{1}\right| & =\frac{1}{\Gamma(\alpha)}\left|D_{0+}^{\alpha-1} \chi\left(t_{1}\right)-\lambda \int_{0}^{t_{1}} f\left(s, x(s), D_{0+}^{\alpha-1} \chi(s)\right) d s\right| \\
& \leqslant \frac{1}{\Gamma(\alpha)}\left(G+\int_{0}^{1}\left|f\left(s, x(s), D_{0+}^{\alpha-1} \chi(s)\right)\right| d s\right) \\
& =\frac{1}{\Gamma(\alpha)}\left(G+\|N x\|_{1}\right),
\end{aligned}
$$

and

$$
\begin{aligned}
\left|c_{2}\right| & =\left|t_{0}^{2-\alpha} \chi\left(t_{0}\right)-\frac{\lambda}{\Gamma(\alpha)} t_{0}^{2-\alpha} \int_{0}^{t_{0}}\left(t_{0}-s\right)^{\alpha-1} f\left(s, x(s), D_{0+}^{\alpha-1} \chi(s)\right) d s-c_{1} t_{0}\right| \\
& \leqslant G+\frac{1}{\Gamma(\alpha)} \int_{0}^{1}\left|f\left(s, x(s), D_{0+}^{\alpha-1} \chi(s)\right)\right| d s+\left|c_{1}\right| \\
& =G+\frac{1}{\Gamma(\alpha)}\|N x\|_{1}+\left|c_{1}\right| .
\end{aligned}
$$

Thus,

$$
\begin{aligned}
\left|\mathrm{t}^{2-\alpha} \chi(\mathrm{t})\right| & \leqslant \frac{1}{\Gamma(\alpha)} \mathrm{t}^{2-\alpha} \int_{0}^{\mathrm{t}}(\mathrm{t}-\mathrm{s})^{\alpha-1}\left|\mathrm{f}\left(\mathrm{s}, \chi(\mathrm{s}), \mathrm{D}_{0+}^{\alpha-1} \chi(\mathrm{s})\right)\right| \mathrm{d} s+\left|\mathrm{c}_{1}\right| \mathrm{t}+\left|\mathrm{c}_{2}\right| \\
& \leqslant \frac{1}{\Gamma(\alpha)} \int_{0}^{1}\left|\mathrm{f}\left(\mathrm{s}, \chi(\mathrm{s}), \mathrm{D}_{0+}^{\alpha-1} \chi(\mathrm{s})\right)\right| \mathrm{d} s+\left|\mathrm{c}_{1}\right|+\left|\mathrm{c}_{2}\right| \\
& \leqslant \frac{4}{\Gamma(\alpha)}\|\mathrm{N} x\|_{1}+\frac{2 \mathrm{G}}{\Gamma(\alpha)}+\mathrm{G} .
\end{aligned}
$$

Therefore,

$$
\begin{aligned}
\|P x\|_{X} & =\max \left\{\left\|t^{2-\alpha} P x\right\|_{\infty},\left\|D_{0+}^{\alpha-1} P x\right\|_{\infty}\right\} \\
& \leqslant \delta\left|\lim _{t \rightarrow 0^{+}} t^{2-\alpha} x(t)\right| \leqslant \delta\left(\frac{4}{\Gamma(\alpha)}\|N x\|_{1}+\frac{2 G}{\Gamma(\alpha)}+G\right) .
\end{aligned}
$$

Also, for $x \in \Omega_{1}, x \in \operatorname{domL} \backslash \operatorname{KerL}$, then $(\mathrm{I}-\mathrm{P}) x \in \operatorname{domL} \cap \operatorname{KerP}, \mathrm{LP} x=0$, from Lemma 3.3, we have

$$
\begin{aligned}
\|(\mathrm{I}-\mathrm{P}) \mathrm{x}\|_{\mathrm{X}} & =\left\|\mathrm{K}_{\mathrm{p}} \mathrm{L}(\mathrm{I}-\mathrm{P}) \mathrm{x}\right\|_{\mathrm{X}} \leqslant \Delta\|\mathrm{L}(\mathrm{I}-\mathrm{P}) x\|_{1} \\
& =\Delta\|\mathrm{L} x\|_{1} \leqslant \Delta\|\mathrm{N} x\|_{1} .
\end{aligned}
$$

It follows from (3.6) and (3.7) that

$$
\begin{aligned}
\|x\|_{X} & =\|P x+(I-P) x\|_{X} \leqslant\|P x\|_{X}+\|(I-P) x\|_{X} \\
& \leqslant\left(\frac{2 G}{\Gamma(\alpha)}+G\right) \delta+\left(\frac{4 \delta}{\Gamma(\alpha)}+\Delta\right)\|N x\|_{1} .
\end{aligned}
$$


By $(\mathrm{H} 1)$, we have

$$
\begin{aligned}
\|N x\|_{1} & =\int_{0}^{1}\left|f\left(s, x(s), D_{0+}^{\alpha-1} x(s)\right)\right| d s \\
& \leqslant \int_{0}^{1}\left[s^{2-\alpha}\left|p(s)\|x(s)|+| q(s)\| D_{0+}^{\alpha-1} \chi(s)\right|+|r(s)|\right] d s \\
& \leqslant\|x\|_{X}\left(\|p\|_{1}+\|q\|_{1}\right)+\|r\|_{1} .
\end{aligned}
$$

Substitute (3.9) into (3.8), one gets,

$$
\|x\|_{X} \leqslant \frac{(2+\Gamma(\alpha)) G \delta+\|r\|_{1}(4 \delta+\Gamma(\alpha) \Delta)}{\Gamma(\alpha)-(4 \delta+\Gamma(\alpha) \Delta)\left(\|p\|_{1}+\|q\|_{1}\right)} .
$$

That is, $\Omega_{1}$ is bounded.

Lemma 3.6. Suppose that (H3) holds, set

$$
\Omega_{2}=\{x \in \operatorname{Ker} \mathrm{L}: \mathrm{N} x \in \operatorname{ImL}\} .
$$

Then, $\Omega_{2}$ is bounded.

Proof. For $x \in \Omega_{2}$, then $x$ can be rewritten as $x=c[1+(\rho-1) t] t^{\alpha-2}, c \in \mathbb{R}$ and $Q N x=0$. From (H3), we get $|c| \leqslant M$. Then, we have

$$
\left\|D_{0+}^{\alpha-1} x\right\|_{\infty}=|c(\rho-1)| \Gamma(\alpha) \leqslant M \delta \Gamma(\alpha),
$$

and

$$
\left\|x_{\alpha}\right\|_{\infty}=\left\|t^{2-\alpha} \chi(t)\right\|_{\infty}=\|c+c(\rho-1) t\|_{\infty} \leqslant M(1+\delta),
$$

which implies that $\Omega_{2}$ is bounded.

Lemma 3.7. Suppose that (H3) holds, set

$$
\Omega_{3}=\{x \in \operatorname{Ker} L: \vartheta \lambda J x+(1-\lambda) \mathrm{QN} x=0, \lambda \in[0,1]\},
$$

where $\mathrm{J}: \mathrm{Ker} \mathrm{L} \rightarrow \operatorname{ImQ}$ is the linear isomorphism defined by

$$
J\left\{c[1+(\rho-1) t] t^{\alpha-2}\right\}=c, \quad \forall c \in \mathbb{R} .
$$

Then $\Omega_{3}$ is bounded where $\vartheta=1$, if (3.4) holds and $\vartheta=-1$ if (3.5) holds.

Proof. For $x=c[1+(\rho-1) t] t^{\alpha-2} \in \Omega_{3}$, without loss of generality, we suppose that (3.4) holds, then there exists $\lambda \in[0,1]$ such that

$$
\lambda c+(1-\lambda) \mathrm{QN}\left\{\mathrm{c}[1+(\rho-1) \mathrm{t}] \mathrm{t}^{\alpha-2}\right\}=0 .
$$

If $\lambda=1$, then $|c|=0 \leqslant M$. Otherwise, if $|c|>M$, by (3.4) we have

$$
\lambda c^{2}=-(1-\lambda) \operatorname{cQN}\left\{c[1+(\rho-1) t] t^{\alpha-2}\right\}<0,
$$

which is a contradiction. So, $\Omega_{3}$ is bounded.

Theorem 3.8. Suppose that (H0)-(H3) hold. Then the problem (1.1) has at least one solution in X.

Proof. Set $\Omega$ be a bounded open set of $X$ such that $\cup_{i=1}^{3} \bar{\Omega}_{i} \subset \Omega$. By Lemma 3.4, N is L-compact on $\bar{\Omega}$. From Lemmas 3.5 and 3.6, we get

(i) $L x \neq \lambda \mathrm{N} x$ for every $(x, \lambda) \in[(\operatorname{dom} L \backslash \operatorname{KerL}) \cap \partial \Omega] \times(0,1)$; 
(ii) $\mathrm{N} x \in \operatorname{ImL}$ for every $x \in \operatorname{KerL} \cap \partial \Omega$.

In the following, we only need to check (iii) of Lemma 2.1 is satisfied. Take,

$$
\mathrm{H}(x, \lambda)=\vartheta \lambda \mathrm{J} x+(1-\lambda) \mathrm{QN} x,
$$

where $\vartheta=1$, if (3.4) holds and $\vartheta=-1$, if (3.5) holds. According to Lemma 3.7, we derive $H(x, \lambda) \neq 0$ for all $x \in \operatorname{KerL} \cap \partial \Omega$. Thus, it follows from the homotopy of degree that

$$
\begin{aligned}
\operatorname{deg}\left\{\left.\mathrm{QN}\right|_{\operatorname{KerL}^{\prime}}, \Omega \cap \operatorname{KerL}, 0\right\} & =\operatorname{deg}\{\mathrm{H}(\cdot, 0), \Omega \cap \operatorname{KerL}, 0\} \\
& =\operatorname{deg}\{\mathrm{H}(\cdot, 1), \Omega \cap \operatorname{KerL}, 0\} \\
& =\operatorname{deg}\{\vartheta], \Omega \cap \operatorname{KerL}, 0\} \neq 0 .
\end{aligned}
$$

Therefore, by Lemma 2.1 we can get that operator function $L x=N x$ has at least one solution in domL $\cap \bar{\Omega}$, which is equivalent to problem (1.1) has at least one solution in $\mathrm{X}$.

\section{Example}

Example 4.1. Consider the boundary value problems

$$
\left\{\begin{array}{l}
D_{0+}^{3 / 2} x(t)=\sin t+\frac{1}{15} t^{1 / 2} \sin x(t)+\frac{1}{30}\left(t^{1 / 2}|x(t)|+\left|D_{0+}^{1 / 2} x(t)\right|\right), \quad t \in(0,1), \\
\lim _{t \rightarrow 0^{+}} t^{1 / 2} x(t)=\frac{\sqrt{2}}{2} \chi(1 / 2), x(1)=\frac{1}{2} \int_{0}^{1} x(t) d t
\end{array}\right.
$$

where we take

$$
\begin{gathered}
A(t)=\left\{\begin{array}{l}
0, \quad t \in[0,1 / 2), \\
\sqrt{2} / 2, \quad t \in[1 / 2,1], \quad B(t)=\frac{1}{2} t, \quad \alpha=\frac{3}{2},
\end{array}\right. \\
f\left(t, x(t), D_{0+}^{\alpha-1} x(t)\right)=\sin t+\frac{1}{15} t^{1 / 2} \sin x(t)+\frac{1}{30}\left(t^{1 / 2}|x(t)|+\left|D_{0+}^{1 / 2} x(t)\right|\right) .
\end{gathered}
$$

Then, we have

$$
\lim _{t \rightarrow 0^{+}} t^{2-\alpha} x(t)=\int_{0}^{1} x(t) d A(t)=\frac{\sqrt{2}}{2} x(1 / 2), \quad x(1)=\int_{0}^{1} x(t) d B(t)=\frac{1}{2} \int_{0}^{1} x(t) d t .
$$

Thus, (4.1) can be as an example of boundary value problem (1.1). By (4.2) and (4.3) we can derive

$$
\begin{gathered}
\left|f\left(t, x(t), D_{0+}^{\alpha-1} \chi(t)\right)\right| \leqslant \frac{1}{10} t^{1 / 2}|x(t)|+\frac{1}{30}\left|D_{0+}^{1 / 2} x(t)\right|+1, \\
\Lambda_{1}=1-\int_{0}^{1} t^{\alpha-2}(1-t) d A(t)=\frac{1}{2}, \quad \Lambda_{2}=\int_{0}^{1} t^{\alpha-1} d A(t)=\frac{1}{2}, \\
\Lambda_{3}=\int_{0}^{1} t^{\alpha-2}(1-t) d B(t)=\frac{2}{3}, \quad \Lambda_{4}=1-\int_{0}^{1} t^{\alpha-1} d B(t)=\frac{2}{3}, \\
\rho=\Lambda_{3} / \Lambda_{4}=\Lambda_{1} / \Lambda_{2}=1, \delta=\max \{1,|\rho|,|\rho-1|\}=1, \Delta=\frac{2}{\Gamma(\alpha)}+\frac{\left|\int_{0}^{1} t^{\alpha-1} d\right| B(t) \mid}{\Gamma(\alpha)\left|\Lambda_{4}\right|}=\frac{5}{\sqrt{\pi}^{\prime}} \\
\Lambda=\frac{\Lambda_{3}}{\Gamma(\alpha+1)} \int_{0}^{1} t^{\alpha-1}(1-t) d A(t)+\frac{\Lambda_{1}}{\Gamma(\alpha+1)} \int_{0}^{1} t^{\alpha-1}(1-t) d B(t)=\frac{14}{45 \sqrt{\pi}} \neq 0 .
\end{gathered}
$$

Let, $p(t) \equiv \frac{1}{10}, q(t) \equiv \frac{1}{30}, r(t) \equiv 1$, then

$$
\|p\|_{1}+\|q\|_{1}=\frac{2}{15}<\frac{\sqrt{\pi}}{13}=\frac{\Gamma(\alpha)}{4 \delta+\Gamma(\alpha) \Delta} .
$$


Therefore, the conditions (H0) and (H1) hold.

Now, we show that the conditions (H2) and (H3) hold. Take $G=M=40$, we easily check for $t^{1 / 2}|x(t)|>40$ or $\left|D_{0+}^{1 / 2} x(t)\right|>40$, then $f\left(t, x(t), D_{0+}^{1 / 2} x(t)\right)>0$. According to the definition of $g(t, s)$, we can get $g(t, s) \geqslant 0$, thus

$$
\begin{aligned}
\mathrm{QN}(x(\mathrm{t})) & =\frac{\Lambda_{3}}{\Lambda} \int_{0}^{1} \int_{0}^{1} g(t, s) f\left(s, x(s), D_{0+}^{1 / 2} \chi(s)\right) d s d A(t)+\frac{\Lambda_{1}}{\Lambda} \int_{0}^{1} \int_{0}^{1} g(t, s) f\left(s, x(s), D_{0+}^{1 / 2} \chi(s)\right) d s d B(t) \\
& =\frac{\sqrt{2} \Lambda_{3}}{2 \Lambda} \int_{0}^{1} g(1 / 2, s) f\left(s, x(s), D_{0+}^{1 / 2} \chi(s)\right) d s+\frac{\Lambda_{1}}{2 \Lambda} \int_{0}^{1} \int_{0}^{1} g(t, s) f\left(s, x(s), D_{0+}^{1 / 2} \chi(s)\right) d s d t \\
& \geqslant \frac{\sqrt{2} \Lambda_{3}}{2 \Lambda} \int_{0}^{1} g(1 / 2, s) f\left(s, x(s), D_{0+}^{1 / 2} \chi(s)\right) d s \\
& =\frac{\sqrt{2} \Lambda_{3}}{\sqrt{\pi} \Lambda} \int_{0}^{1 / 2}\left[(1 / 2-s / 2)^{1 / 2}-(1 / 2-s)^{1 / 2}\right] f\left(s, x(s), D_{0+}^{1 / 2} \chi(s)\right) d s \\
& +\frac{\Lambda_{3}}{\sqrt{\pi} \Lambda} \int_{1 / 2}^{1}(1-s)^{1 / 2} f\left(s, x(s), D_{0+}^{1 / 2} x(s)\right) d s \\
& \geqslant \frac{\Lambda_{3}}{\sqrt{\pi} \Lambda} \int_{1 / 2}^{1}(1-s)^{1 / 2} f\left(s, x(s), D_{0+}^{1 / 2} x(s)\right) d s>0 .
\end{aligned}
$$

In addition, when $|c|>40$, we have $N\left\{c[1+(\rho-1) t] t^{\alpha-2}\right\}=N\left(c t^{-1 / 2}\right)=f\left(t, c t^{-1 / 2}, 0\right)>0$, that is, the conditions (H2) and (H3) hold. By Theorem 3.8, problem (4.1) has at least one solution.

\section{Acknowledgment}

This research is supported by the National Natural Science Foundation of China (No. 11271364).

\section{References}

[1] B. Ahmad, S. K. Ntouyas, Existence results for higher-order fractional differential inclusions with Riemann-Stieltjes type integral boundary conditions, Commun. Appl. Anal., 17 (2013), 87-98. 1

[2] C.-Z. Bai, Impulsive periodic boundary value problems for fractional differential equation involving Riemann-Liouville sequential fractional derivative, J. Math. Anal. Appl., 384 (2011), 211-231. 1

[3] Z.-B. Bai, H.-S. Lü, Positive solutions for boundary value problem of nonlinear fractional differential equation, J. Math. Anal. Appl., 311 (2005), 495-505. 1, 2.4, 2.5, 2.6

[4] A. Cabada, G.-T. Wang, Positive solutions of nonlinear fractional differential equations with integral boundary value conditions, J. Math. Anal. Appl., 389 (2012), 403-411. 1

[5] Y.-J. Cui, Solvability of second-order boundary-value problems at resonance involving integral conditions, Electron. J. Differential Equations, 2012 (2012), 9 pages. 1, 1

[6] H. A. A. El-Saka, The fractional-order SIS epidemic model with variable population size, J. Egyptian Math. Soc., 22 (2014), 50-54. 1

[7] W.-H. Jiang, The existence of solutions to boundary value problems of fractional differential equations at resonance, Nonlinear Anal., 74 (2011), 1987-1994. 1, 2.4, 2.5, 2.6

[8] A. A. Kilbas, H. M. Srivastava, J. J. Trujillo, Theory and applications of fractional differential equations, North-Holland Mathematics Studies, Elsevier Science B.V., Amsterdam, (2006). 1, 2.2, 2.3, 2.4, 2.5

[9] W.-W. Liu, J.-Q. Jiang, L.-S. Liu, Y.-H. Wu, Nontrivial solutions of singular Sturm-Liouville problem with boundary conditions involving Riemann-Stieltjes integrals, Nonlinear Funct. Anal. Appl., 17 (2012), 255-271. 1

[10] R. L. Magin, Fractional calculus models of complex dynamics in biological tissues, Comput. Math. Appl., 59 (2010), 1586-1593. 1

[11] J. Mawhin, Topological degree methods in nonlinear boundary value problems, Expository lectures from the CBMS Regional Conference held at Harvey Mudd College, Claremont, Calif., June 9-15, (1977), CBMS Regional Conference Series in Mathematics, American Mathematical Society, Providence, R.I., (1979). 1, 2, 2.1

[12] J. Mawhin, Topological degree and boundary value problems for nonlinear differential equations, Topological methods for ordinary differential equations (Montecatini Terme, 1991), Lecture Notes in Math., Springer, Berlin, 1537 (1993), 74-142. 1, 2, 2.1 
[13] K. S. Miller, B. Ross, An introduction to the fractional calculus and fractional differential equations, A Wiley-Interscience Publication, John Wiley \& Sons, Inc., New York, (1993). 1, 2.2, 2.3, 2.4, 2.5

[14] I. Podlubny, Fractional differential equationss, An introduction to fractional derivatives, fractional differential equations, to methods of their solution and some of their applications, Mathematics in Science and Engineering, Academic Press, Inc., San Diego, CA, (1999).

[15] J. Sabatier (Ed.), O. P. Agrawal (Ed.), J. A. Tenreiro Machado (Ed.), Advances in fractional calculus, Theoretical developments and applications in physics and engineering, Including papers from the Minisymposium on Fractional Derivatives and their Applications (ENOC-2005) held in Eindhoven, August 2005, and the 2nd Symposium on Fractional Derivatives and their Applications (ASME-DETC 2005) held in Long Beach, CA, September (2005), Springer, Dordrecht, (2007). 1

[16] K. Szymańska-Dębowska, k-dimensional nonlocal boundary-value problems at resonance, Electron. J. Differential Equations, 2015 (2015), 8 pages. 1

[17] Y. Wang, L.-S. Liu, X.-G. Zhang, Y.-H. Wu, Positive solutions of an abstract fractional semipositone differential system model for bioprocesses of HIV infection, Appl. Math. Comput., 258 (2015), 312-324.

[18] X.-G. Zhang, Y.-F. Han, Existence and uniqueness of positive solutions for higher order nonlocal fractional differential equations, Appl. Math. Lett., 25 (2012), 555-560. 1

[19] X.-G. Zhang, L.-S. Liu, Y.-H. Wu, The uniqueness of positive solution for a fractional order model of turbulent flow in a porous medium, Appl. Math. Lett., 37 (2014), 26-33. 1 